\title{
Blood Velocity Estimation Based on an Optimal Observer for Magnetic Nanorobots Navigation
}

\author{
Ton Duc Do \\ Department of Robotics and Mechatronics, School of Science and Technology (SST), Nazarbayev University, 53 KabanbayBatyr Ave, \\ Astana Z05HOP9, Republic of Kazakhstan
}

\begin{abstract}
Magnetic nanorobots are recently used in a drug delivery system in order to perform minimally invasive medical procedures. One main difficulty for controlling nanorobots is the complicated behavior of hydrodynamic drag force and uncertain factors in their dynamics. The drag force depends on the blood velocity but unfortunately, it is apparently unknown. In previous feedback control system designs, the blood velocity was assumed to be known or set constant. This assumption did not reflect the reality and reduce the feasibility of the control systems. Considering this fact, this paper suggests an optimal observer for estimating blood velocity. The only information required for the proposed observer and overall control system is the position of nanorobots. The characteristic, stability, as well as gain tuning rules of the proposed optimal observer is discussed in detail. Simulation results are carried out in MATLAB/Simulink to demonstrate the effectiveness of the proposed optimal observer as well as the overall feedback control systems for nanorobot navigation in blood vessels.
\end{abstract}

\section{Introduction}

Recently, minimally invasive medical treatments have incredible growth since related techniques can approach the remote sites without surgical operation and improve targeting. To achieve these objectives, an interesting technique called magnetic drug targeting (MDT) that adds drugs to magnetic nanorobots and then applies external magnetic fields to steer and concentrate them at disease locations is used. Typically, the circulation of the blood will spread the drug throughout the body that may cause systemic toxicity in organs to which the drug is not targeted. Magnetic drug targeting is the desired technique to maximize the therapeutic efficiency and safety while avoiding medical side effects in healthy tissues [1]-[4]

There are two mains approaches in MDT that is opened-loop control [5], [6] and closed-loop control [7][14]. Each technique has its own advantages and drawbacks. The opened-loop control approach does not require any feedback information such as position of nanorobots, however, its efficiency is affected by a lot of factors such as vessel geometry, blood velocity, and magnetic field gradient as defined in [5]. Complicated mutual relations of these parameters need to be carefully considered or it will result in low efficiency [6]. One main disadvantage of closed-loop control approach is the requirement of real-time feedback information of nanorobots position. Fortunately, nowadays, this information can be achieved with monitoring devices such as magnetic resonance imaging (MRI) or magnetic particle imaging (MPI). Another drawback of most published closed-loop control methods [7]-[13] is that they only deal with aggregated particles in which the effective radii are about a few hundred micrometers. However, with the ability of navigating nanorobots to the correct sites precisely and effectively, closed-loop control techniques are a promising approach.

In all feedback control system designs, nanorobots depend on different forces whose modelling is vital to predict their behavior in blood vessels. However, the blood velocity, which is the key parameter of the drag force, is unknown. The measurement of blood velocity is a challenged task since it requires ultrasonic sensors. An alternative is using computational fluid dynamics software to achieve the numerical resolution of the blood velocity [15], [16]. But, this technique requires a lot of computation effort since it is based on Navier-Stokes equations. Therefore, it is not feasible for real-time applications such as in feedback control systems. The authors in [17] introduced analytical expressions of the blood velocity profiles near the bifurcation. However, the vessel geometry information is essential. In conclusion, online estimation of the blood velocity seems to be the most appropriate approach for avoiding the aforementioned difficulties. In [14], two observers are suggested for estimating the blood velocity. The simulation results show that these observers can precisely estimate the blood velocity and significantly enhanced the control performance.

This paper introduces an optimal observer for estimating blood velocity as an input to the adaptive sliding-mode control (ASMC) system. The stability and 
gain tuning rules of the proposed observer are discussed in detail. The numerous simulation results show that the proposed optimal observer can precisely estimate the blood. Simulations also prove that the proposed observerbased control system can attain high-performance under different scenarios.

\section{Problem formulations}

The state-space dynamic modelling of aggregate nanorobots in arterials [11]-[14] is as follows,

$$
\left\{\begin{array}{c}
\dot{x}_{1}=x_{2} \\
\dot{x}_{2}=f\left(x_{2}, v_{1}\right)+\frac{\tau_{m} M}{\rho} u \\
y=x_{1}
\end{array}\right.
$$

where $x_{1}$ and $x_{2}$ are the aggregate position and velocity, respectively, $u$ is the magnetic field gradient considered as control input, $v_{1}$ is the blood velocity, $\tau_{m}$ is the ferromagnetic ratio, $\rho$ is the aggregate density, $M$ is the magnetization, and $y$ is the output which is the aggregate position measured by MRI or MPI, respectively. The function $f$ is expressed as,

$$
\begin{aligned}
& f\left(x_{2}, v_{1}\right)=\frac{1}{m}\left[-\operatorname{sgn}\left(x_{2}-v_{1}\right)\left(\left(a\left|x_{2}-v_{1}\right|\right)\right.\right. \\
& +b\left(x_{2}-v_{1}\right)^{2}+c \frac{\left(x_{2}-v_{1}\right)^{2}}{1+d \sqrt{\left|x_{2}-v_{1}\right|}} \\
& \left.\left.+V\left(\rho_{f}-\rho\right) g\right)\right]
\end{aligned}
$$

where

$$
\begin{gathered}
a=\frac{6 \pi \eta r}{\beta}, b=\frac{0.2 \rho f \pi r^{2}}{\beta^{2}}, c=\frac{3 \rho f \pi r^{2}}{\beta^{2}}, d=\sqrt{\frac{2 r \rho_{f}}{\beta \eta}}, \\
\operatorname{sgn}(z)=\left\{\begin{array}{l}
-1 \text { if } z<0 \\
0 \text { if } z=0 \\
1 \text { if } z>0
\end{array}\right.
\end{gathered}
$$

In (3), $r$ is the aggregate radius, $\beta$ is a dimensionless ratio related to the wall effect caused by the vessel occlusion by a spherical aggregate of radius $r, \eta$ and $\rho_{f}$ are the blood viscosity and density, respectively.

Note that the function $f$ in (1) and (2) includes the expression of drag force and weight (the last term in the bracket is weight and the others are drag force) whereas the control input $u$ is proportional the magnetic force. Also, in (1), only the nanorobot position $x_{1}$ is measurable, the nanorobot velocity and blood velocity are needed to be calculated and estimated.

In order to estimate the blood velocity, its dynamic equation should be clarified. According to [13], [15], the pulsatile blood velocity $v_{1}$ can be defined as an $n$ th-order truncated Fourier series,

$$
\left\{\begin{array}{c}
\dot{v}_{1}=v_{2} \\
\dot{v}_{2}=-\omega^{2}\left(v_{1}-v_{3}\right) \\
\vdots \quad \dot{v}_{2 k-1}=v_{2 k} \\
\dot{v}_{2 k}=-\omega^{2}\left(k^{2} v_{2 k-1}-v_{2 k+1}\right) \\
\vdots \\
\dot{v}_{2 n+1}=0
\end{array}\right.
$$

where $\omega$ is the pulsation of the blood flow assumed to be known and $v_{2 n+1}$ is the average value of the blood velocity.

\section{Feedback control design}

\subsection{Adaptive sliding-mode control design}

Let us consider the following ASMC law for the system (1),

$$
\left\{\begin{array}{l}
\sigma=e_{1}+\alpha e_{2} \\
u=-\frac{1}{\alpha} \frac{\rho}{\tau_{m} M}\left(\alpha f-\dot{x}_{1 r}-\alpha \ddot{x}_{1 r}+\theta \operatorname{sgn}(\sigma)\right) \\
\dot{\theta}=|\sigma| / \gamma
\end{array}\right.
$$

where $e_{1}=x_{1}-x_{1 r}$ and $e_{2}=x_{2}-\dot{x}_{1 r}$ are the tracking errors, $x_{1 r}$ is the position reference command of the nanorobots, $\alpha$ and $\gamma$ are the control gains. In (5), the adaptive control gain $\theta$ is online estimate by an adaptive law to avoid high control gains as well as with high control efforts. The SMC law in (5) may include chattering due to using a sign function. There are several ways to reduce the chattering, however, a simple method is the approximated sign function by the following approximated function:

$$
\operatorname{sgn}(\sigma)=\frac{\sigma}{|\sigma|+\varepsilon}
$$

where $\varepsilon$ is an very small positive number.

\subsection{Nanorobot velocity calculation}

As the nanorobot velocity is the time-derivative of the nanorobot position, it can be directly calculated from nanorobot position by the following discrete-time formula [16]-[18]:

$$
x_{2}=\frac{\varphi}{T+\varphi} x_{2}(k-1)+\frac{1}{T+\varphi}\left[x_{1}(k)-x_{1}(k-1)\right]
$$

where $k$ and $k-1$ denote two consecutive sampling instants, $T$ is the sampling period, and $\varphi$ is a sufficiently small time constant.

\section{Blood velocity observer design}

Combining (1) and (4), we achieve the following dynamic equations for estimating blood velocity, 


$$
\left\{\begin{array}{c}
\dot{x}_{1}=x_{2} \\
\dot{x}_{2}=-a x_{2}+a x_{3}+f^{\prime}\left(x_{2}, x_{3}, u\right) \\
\dot{x}_{3}=x_{4} \\
\dot{x}_{4}=-\omega^{2}\left(x_{3}-x_{5}\right) \\
\vdots \quad \\
\dot{x}_{2 k+1}=x_{2 k+2} \\
\dot{x}_{2 k+2}=-\omega^{2}\left(k^{2} x_{2 k+1}-v_{2 k+3}\right) \\
\vdots \\
\dot{x}_{2 n+3}=0 \quad \\
y=x_{1}
\end{array}\right.
$$

where $x_{i+2}=v_{i} \forall i \in\{1,2, \cdots n\}$ and

$$
\begin{aligned}
f^{\prime}\left(x_{2}, v_{1}, u\right)=\frac{1}{m}[ & -\operatorname{sgn}\left(x_{2}-v_{1}\right)\left(b\left(x_{2}-v_{1}\right)^{2}\right. \\
& \left.\left.+c \frac{\left(x_{2}-v_{1}\right)^{2}}{1+d \sqrt{\left|x_{2}-v_{1}\right|}}+V\left(\rho_{f}-\rho\right) g\right)\right] \\
& +\frac{\tau_{m} M}{\rho} u
\end{aligned}
$$

Equation (8) can be rewritten in the following statespace form,

$$
\left\{\begin{array}{c}
\dot{x}=A x+B f^{\prime} \\
y=x_{1}
\end{array}\right.
$$

where $x=\left[\begin{array}{lll}x_{1} & \ldots & x_{2 n+3}\end{array}\right]^{T}, B=\left[\begin{array}{lllll}0 & 1 & 0 & \ldots & 0\end{array}\right]^{T}$,

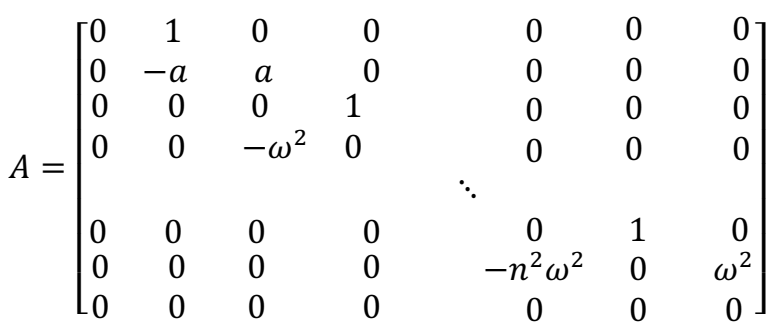

Then, the optimal observer model for estimating blood velocity can be represented as

$$
\left\{\begin{array}{c}
\dot{\hat{x}}=A \hat{x}+L y-L C \hat{x}+B f^{\prime} \\
y=C x \\
\hat{x}_{3}=C_{T} \hat{x}
\end{array}\right.
$$

where

$C=\left[\begin{array}{llll}1 & 0 & \ldots & 0\end{array}\right]^{T}, C_{T}=\left[\begin{array}{llllll}0 & 0 & 1 & 0 & \ldots & 0\end{array}\right]^{T}, \hat{x}$ is the estimated vector of $x$, and $L$ is the observer gain vector.

Fig. 1 illustrates the block diagram of the proposed blood velocity observer.

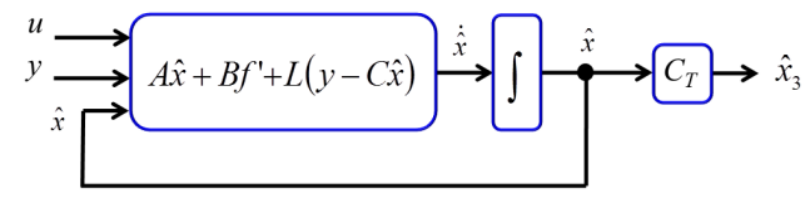

Figure 1. Block diagram of the proposed optimal blood velocity observer.

The error dynamics of the load current observer can be obtained as follows:

$$
\dot{\tilde{x}}=(A-L C) \tilde{x}
$$

where $\tilde{x}=x-\hat{x}$.

Theorem 1: Consider the following algebraic Riccati equation (ARE)

$$
A P+P A^{T}-P C^{T} R^{-1} C P+Q=0
$$

where $Q \in R^{(2 n+3) \times(2 n+3)}$ is a symmetric positive semidefinite matrix, $R$ is a positive number, and $P \in R^{(2 n+3) \times 1}$ is a solution vector. And the load current observer gain matrix $L$ is given by

$$
L=P C^{T} R^{-1}
$$

Then, the estimation error converges exponentially to zero.

Proof: Consider the following Lyapunov function: $V(\tilde{x})=\tilde{x}^{T} H \tilde{x}$, where $H=P^{-1}$. Its time derivative along the error dynamics (10) is given by

$$
\begin{aligned}
\dot{V}=\frac{d}{d t} \tilde{x}^{T} H \tilde{x}= & 2 \tilde{x}^{T}\left(H A-P C^{T} R^{-1} C\right) \tilde{x} \\
& =\tilde{x}^{T} H\left(A P+P A^{T}-2 P C^{T} R^{-1} C P\right) H \tilde{x} \\
& \leq-\tilde{x}^{T} H Q H \tilde{x}
\end{aligned}
$$

This implies that $\tilde{x}$ is exponentially stable.

Remark 1: The proposed blood velocity observer is the Kalman-Bucy optimal observer which minimizes the performance index $E\left(\tilde{x}^{T} \tilde{x}\right)$ representing the expectation value of $\tilde{x}^{T} \tilde{x}$ for the following perturbed model

$$
\left\{\begin{array}{l}
\dot{x}=A x+B f^{\prime}+d \\
y=C x+v
\end{array}\right.
$$

where $d \in R^{2 n+3}, \quad v$ is a scalarare independent white Gaussian noise signals with $E(d)=0, E(v)=0, E\left(d d^{T}\right)=$ $Q$, and $E\left(v^{2}\right)=R$.

Remark 2: Typically, the observer performance is mostly influenced by the system model if the measurements are noisy ( $R$ large) and the input noise intensity is small ( $Q$ small). Therefore, $L$ is small. This leads to a slow observer as measured by the location of its eigenvalues. However, if the measurements are good and the input noise intensity is large, the observer relies on the measurement. In this case, $L$ is large, resulting in a fast observer with high bandwidth. Consequently, by assuming that the measurement is good, the fast observer is desirable. Lastly, the subsequent procedure summarizes the tuning process of the observer gain matrix $L[19]$ :

1) $Q$ and $R$ are set as identity matrices;

2) Gradually, increase $Q$ and decrease $R$, then calculate

$L$ as in (12) and (13);

If the observer performance is not satisfied, return to step 2 above. Otherwise, quit.

\section{Results and discussion}

Referring to [11]-[14], the modelling parameters are summarized in Table I. Without loss of generality, we consider the blood velocity as a second-order truncated Fourier series. In this paper, the angular frequency of the blood velocity is chosen as $\omega=6.28 \mathrm{rad} / \mathrm{s}$. With the guidelines in Remark 2 and via extensive simulation trials, 
the gains of controller and weighting matrices of observer are selected as follows:

$$
\begin{gathered}
\alpha=100, \gamma=0.2, \varepsilon=0.001, \\
Q=\operatorname{diag}(1,1000,1,1,1), \\
R=1
\end{gathered}
$$

Then the observer gain matrix are achieve as follows,

$$
L=\left[\begin{array}{lllll}
8.016 & 31.622 & 0.7807 & -8.838 & 1.00
\end{array}\right]^{T}
$$

Table I. Modelling parameters.

\begin{tabular}{llll}
\hline Parameter & Notation & Value & Unit \\
\hline Blood density & $\rho_{f}$ & 1060 & $\mathrm{~kg} / \mathrm{m}^{3}$ \\
\hline Blood viscosity & $\eta$ & 0.0016 & $\mathrm{~Pa} . \mathrm{s}$ \\
\hline Aggregate radius & $r$ & $2.5 \times 10^{-4}$ & $\mathrm{~m}$ \\
\hline Payload density & $\rho_{p}$ & 1500 & $\mathrm{~kg} / \mathrm{m}^{3}$ \\
\hline $\begin{array}{l}\text { Ferromagnetic } \\
\text { ratio }\end{array}$ & $\tau_{m}$ & 0.75 & - \\
\hline nanorobot density & $\rho_{m}$ & 7500 & $\mathrm{~kg} / \mathrm{m}^{3}$ \\
\hline Vessel diameter & $D$ & 3 & $\mathrm{~mm}$ \\
\hline Magnetization & $M$ & 1230 & $\mathrm{kA} / \mathrm{m}$ \\
\hline
\end{tabular}
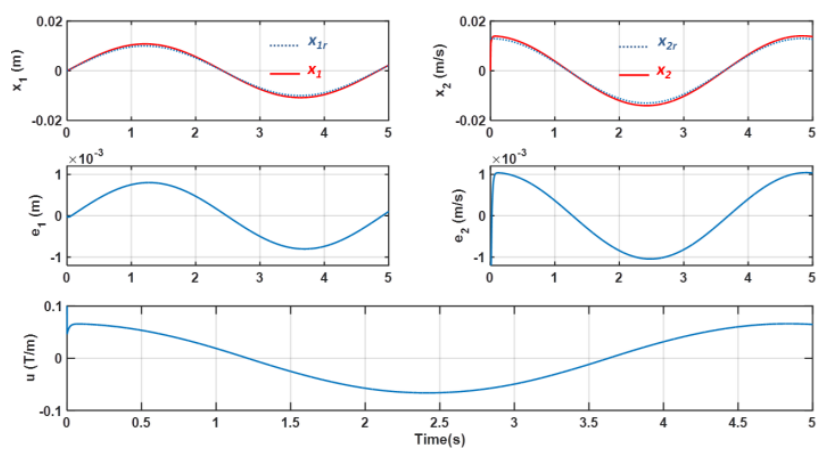

(a) References and real trajectories of nanorobot $\left(x_{1 r}\right.$ in dotted blue and $x_{1}$ in red), real and calculated velocity of nanorobot $\left(x_{2 r}\right.$ in dotted blue and $x_{2}$ in red), position tracking error $\left(e_{1}\right)$, nanorobot velocity calculation error $\left(e_{2}\right)$, and control input magnetic field gradient $(u)$.
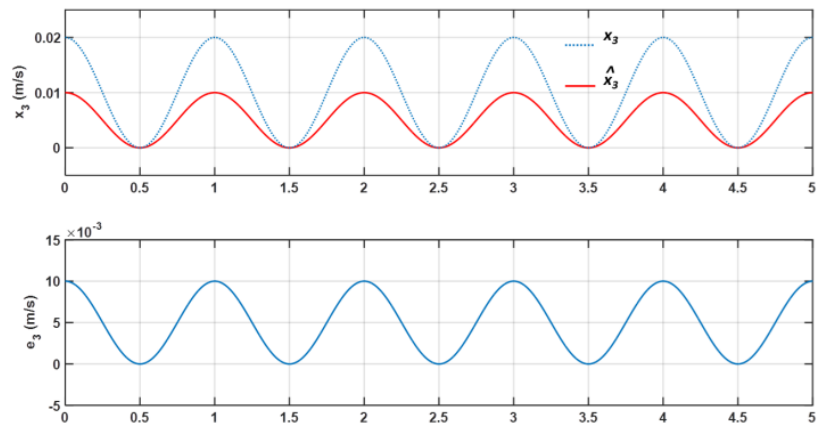

(b) Real and estimated blood velocities ( $x_{3}$ in dotted blue and $\hat{x}_{3}$ in red) and estimation error $\left(e_{3}\right)$

Figure 2. Simulation results without a blood velocity observer.

Fig. 2 shows the simulation results of the ASMC scheme without a blood velocity observer with a $50 \%$ of estimation error whereas Figs. 3 and 4 illustrate the simulation results with the proposed blood velocity observer. Also Figs. 2 and 3 are attained with the aggregate nanorobots with the radius size equal to $2.5 \times 10^{-4} \mathrm{~m}$ whereas Fig. 4 is achieved with single nanorobot with radius of $400 \times 10^{-9}$ m. Figs. 2-4 (a) demonstrates the references and real trajectories $\left(x_{1 r}\right.$ and $\left.x_{1}\right)$, real and calculated velocity of nanorobot $\left(x_{2 r}\right.$ and $\left.x_{2}\right)$, tracking error $\left(e_{1}\right)$, nanorobot velocity calculation error $\left(e_{2}\right)$, and control input - magnetic field gradient $(u)$. And Figs. 2-4 (b) shows real and estimated velocities $\left(x_{3}\right.$ and $\left.\hat{x}_{3}\right)$ and estimation error $\left(e_{3}\right)$. Note that only the real velocity of nanorobot $x_{2 r}$, calculated nanorobot velocity $x_{2}$ and estimated blood velocity $\hat{x}_{3}$ are available, the blood velocity $x_{3}$ are not available; however, in simulations, we show $x_{3}$ in order to verify the effectiveness of the proposed optimal observer.

In Fig. 2, it should be noticed that the position tracking error without an observer is quite large $(0.85 \mathrm{~mm})$ and limit the access to the desired site of human body. Also it may not be feasible to control with such a big tracking error since the blood vessel diameter is just 3 $\mathrm{mm}$. Whereas in Fig. 3, the estimated blood velocity is converge very fast $(1.1 \mathrm{~ms})$ with satisfied estimation error $(0.19 \mathrm{~mm} / \mathrm{s})$

By comparing the results from Figs. 2 and 3, we can see that with the proposed observer, the blood velocity is accurately estimated (the estimation error is $0.19 \mathrm{~mm} / \mathrm{s}$ ) and consequently the better position tracking error and nanorobot velocity calculation error are attained (proposed observer/without observer: position tracking error $0.85 / 0.079[\mathrm{~mm}]$, nanorobot velocity calculation error: $1.03 / 0.95[\mathrm{~mm} / \mathrm{s}])$.
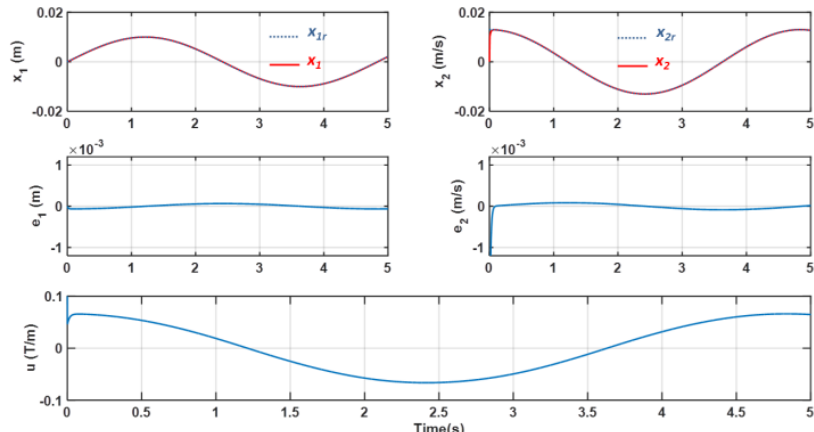

(a) References and real trajectories $\left(x_{1 r}\right.$ in dotted blue and $x_{1}$ in red), real and calculated velocity of nanorobot $\left(x_{2}\right.$ in dotted blue and $x_{2}$ in red), position tracking error $\left(e_{1}\right)$, nanorobot velocity calculation error $\left(e_{2}\right)$, and control input - magnetic field gradient $(u)$.
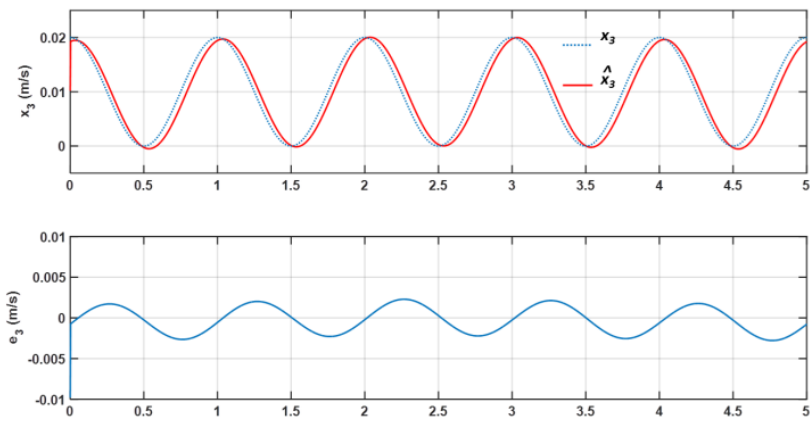

(b) Real and estimated velocities $\left(x_{3}\right.$ in dotted blue and $\hat{x}_{3}$ in red) and estimation error $\left(e_{3}\right)$

Figure 3. Simulation results with proposed blood velocity observer.

In order to further verify the feasibility of the proposed blood velocity observer and overall control scheme, in Fig. 4, we show the simulation results with reduced size of aggregation, i.e. we reduce the size of the object from hundreds micrometer to hundreds nanometer. The 
aggregate radius now is assumed to be $400 \mathrm{~nm}$. Under the same gains as the previous case, in this case, the position tracking error is still in acceptable range whereas the estimation error $(0.21 \mathrm{~mm} / \mathrm{s})$ is almost the same as previous case. Note that the control effort $(2.8 \mathrm{~T} / \mathrm{m})$ is higher compared to the previous case. As presented in [14], an electromagnetic is able to comfortably generate this magnetic field gradient. Also with this result, we prove the proposed blood velocity observer as well as overall control scheme is effective in a wide range of the size of the aggregate nanorobots. In other words, the limitation indicated in [13] is solved.
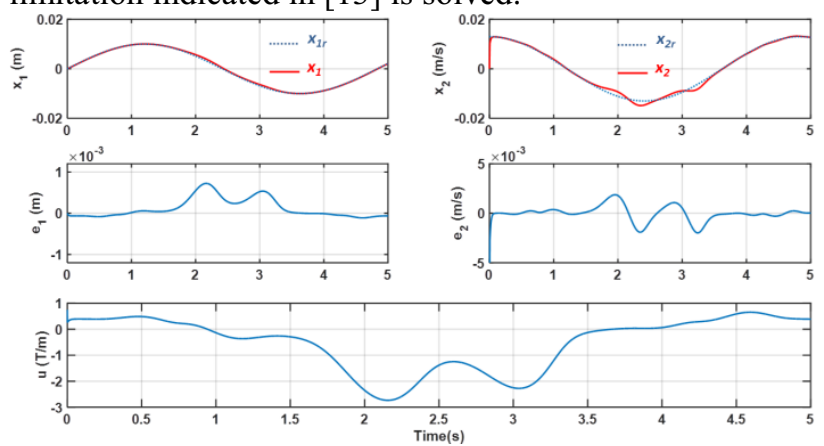

(a) References and real trajectories $\left(x_{1}\right.$ in dotted blue and $x_{1}$ in red), real and calculated velocity of nanorobot $\left(x_{2}\right.$ in dotted blue and $x_{2}$ in red), position tracking error $\left(e_{1}\right)$, nanorobot velocity calculation error $\left(e_{2}\right)$, and control input - magnetic field gradient $(u)$.
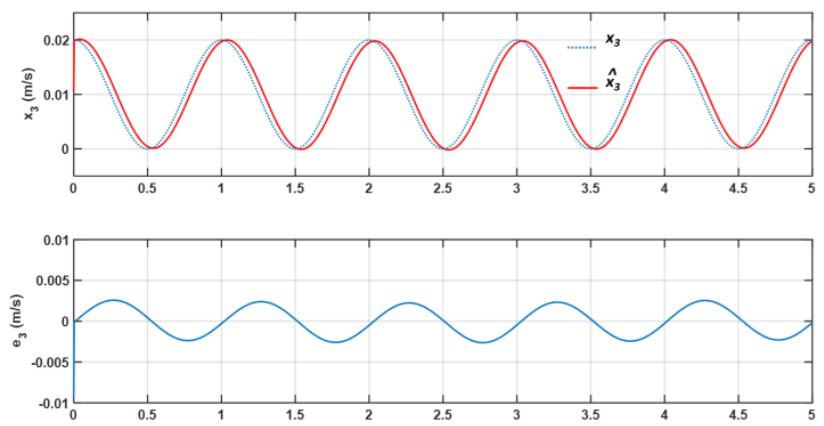

(b) Real and estimated blood velocities ( $x_{3}$ in dotted blue and $\hat{x}_{3}$ in red) and estimation error $\left(e_{3}\right)$

Figure 4. Simulation results with proposed blood velocity observer with single nanorobot.

\section{Conclusions}

In this work, we proposed an optimal observer for blood velocity estimation in blood vessels. Based on this observer, the real-time information of blood velocity is estimated precisely. Then this estimated signal was input to the controller to improve the performance of the overall control system. The only measured signal was position of aggregated nanorobot. We analyzed that the proposed optimal observer is asymptotically stable. The gain tuning rules for proposed observer was also detailed in this paper. Finally, simulation results have shown to prove the effectiveness of the proposed observer as well as overall feedback control system.

\section{References}

1. J. Bar, R. S. Herbst, A. Onn, ExpertOpin. Drug Deliv., 6, 10, (2009)

2. V. P. Torchilin, Handb. Exp. Pharmacol., 197, (2010)

3. S. Martel, J. Nanopart. 17, 2, (2015)

4. M. Hamdi, A. Ferreira, IEEE Trans. Robot., 30, 1, (2014)

5. M. D. Tehrani, J. H. Yoon, M. O. Kim, J. Yoon, IEEE Trans. Biomed. Eng., 62, 1, (2015)

6. T. D. Do, Y. Noh, M. O. Kim, J. Yoon, IROS 2015, Hamburg, Germany, Sep. 28-Oct. 03, (2015)

7. K. B. Yesin, K. Vollmers, B. J. Nelson, Int. J. Robot. Res., 25, 527-536, (2006)

8. J. B. Mathieu, S. Martel, Biomed. Microdevices, 9 , (2007)

9. S. Martel, O. Felfoul, J. B. Mathieu, A. Chanu, S. Tamaz, M. Mohammadi, M. Mankiewicz, N. Tabatabaei, Int. J. Robot. Res., 28, 9, (2009)

10. P. Pouponneau, J. C. Leroux, G. Soulez, L. Gaboury, S. Martel, Biomat., 32, 13, (2011)

11. K. Belharet, D. Folio, A. Ferreira, IEEE Trans. Biomed. Eng., 60, 4, (2013)

12. L. Arcese, M. Fruchard, A. Ferreira, IEEE Trans. Biomed. Eng., 59, 4, (2012)

13. M. Fruchard, L. Arcese, E. Courtial, IEEE Trans. Robot., 30, 1, (2014)

14. T. D. Do, Y. Noh, M. O. Kim, and J. Yoon, J. Nanosci. Nanotechnol., 16, 6, (2016)

15. J. Womersley, J. Physiol., 127, 3, (1955)

16. J. Astrom, B. Witternmark, Computer-Controlled Systems - Theory and Design (Englewood Cliffs, New Jersey: Prentice-Hall, 1990)

17. O. Vainio, IEEE Trans. Ind. Electron., 46, 5, (1999)

18. T. D. Do, H. H. Choi, J. W. Jung, IEEE/ASME Trans. Mechatronics, 20, 6, (2015)

19. B. Shahian and M. Hassul, Control System Design Using Matlab (Englewood Cliffs, New Jersey: Prentice-Hall Inc., 1993) 